\begin{tabular}{|c|c|}
\hline \multirow[t]{6}{*}{ Citation } & Xin Wang, Julian Stoev, Gregory Pinte, Jan Swevers (2013) \\
\hline & Classical and Modern Methods for \\
\hline & Time-constrained Energy Optimal Motion - \\
\hline & Application to a Badminton Robot \\
\hline & Mechatronics (ICM), 2013 IEEE International Conference, pa ge \\
\hline & 706- 711 \\
\hline Archived version & $\begin{array}{l}\text { Author manuscript: the content is identical to the content of the } \\
\text { published paper, but without the final typesetting by the publisher }\end{array}$ \\
\hline \multirow[t]{3}{*}{ Published version } & http://ieeexplore.ieee.or \\
\hline & /xpls/abs_all.jsp?arnumber=6519128\&ta \\
\hline & $=1$ \\
\hline \multicolumn{2}{|l|}{ Journal homepage } \\
\hline \multirow[t]{2}{*}{ Author contact } & xin.wang@mech.kuleuven.be \\
\hline & phone number $+32(0) 16322222$ \\
\hline \multirow[t]{2}{*}{ IR } & https://lirias.kuleu \\
\hline & en.be/handle/123456789/370265 \\
\hline
\end{tabular}




\section{Classical and Modern Methods for Time-constrained Energy Optimal Motion - Application to a Badminton Robot}

\author{
Julian Stoev* and Gregory Pinte* \\ *Flanders' Mechatronics Technology Centre \\ Celestijnenlaan 300 D, 3001 Leuven, Belgium \\ Email: julian.stoev@fmtc.be \\ gregory.pinte@fmtc.be
}

\author{
Xin Wang ${ }^{\dagger}$ and Jan Swevers ${ }^{\dagger}$ \\ $\dagger$ Department of Mechanical Engineering, \\ KU Leuven, Leuven, Belgium \\ Email: xin.wang@mech.kuleuven.be \\ Jan.Swevers@mech.kuleuven.be
}

\begin{abstract}
Two control approaches are presented to improve the energy efficiency of a robot which has to perform point-topoint motions during a fixed time interval. The first approach is based on a time-optimal servo control algorithm whose parameters are optimized in order to achieve energy efficient behavior. The second approach is a energy-optimal model predictive control approach. The developed approaches are applied to a robot playing badminton. The robot is still able to intercept most of the opponent shuttles on time, while a significant reduction of the energy consumption is demonstrated in both cases.
\end{abstract}

\section{INTRODUCTION}

Time-optimal motion control received large attention both in the academic and the industrial world due to the typical requirement for fast and accurate motions maximizing the system's productivity. Proximate Time-Optimal Servo (PTOS) control [1], [2] is widely used for point-to-point motion control. Recently time-optimal MPC is proposed as an alternative control scheme, where actuator saturation and other non-linear effects can explicitly be taken into account.

Energy-optimal control is also a well researched area with enormous references like [3]-[6]. Due to the increasing energy prices, there is a growing industrial interest in energy efficient systems. Since time- and energy-optimality are two conflicting requirements, multi-objective optimization [11] can be used to find the optimal trade-off between these requirements. Current results indicate that for many industrial machines, energy saving is often possible while preserving the essential productivity of the machine.

In this paper two approaches are presented. The first one is a classical approach, where the settings of an existing PTOS motion controller are adapted for each motion, such that less

\footnotetext{
**This work has been carried out within the framework of projects IWTSBO 80032 (LeCoPro) of the Institute for the Promotion of Innovation through Science and Technology in Flanders (IWT-Vlaanderen). This work also benefits from K.U.Leuven-BOF PFV/10/002 Center-of-Excellence Optimization in Engineering (OPTEC), the Belgian Programme on Interuniversity Attraction Poles, initiated by the Belgian Federal Science Policy Office (DYSCO), K.U.Leuvenś Concerted Research Action GOA/10/11 and G.0377.09 of the Research Foundation-Flanders (FWO-Vlaanderen).The research leading to these results has received funding from the European Union Seventh Framework Program (FP7/2007-2013) under grant agreement n247982-ESTOMAD.
}

energy is consumed. This method is further referred to as Proximate Energy Optimal Servo (PEOS). The optimization objective is to achieve energy-optimality while not exceeding motion time requirements. The main advantage of this approach is its computational simplicity. The second approach is a modern approach, based on on-line optimization, more precisely MPC. This method is further referred to as Energy Optimal MPC (EOMPC). The computational requirements for this approach are more stringent, but measurably better performance is obtained.

Outline of the paper: The paper starts with a brief description of our experimental plant - a badminton robot with existing PTOS motion controller. The energy cost function related to our robot is specified. Then the modified PEOS approach is described, followed by the description of the EOMPC. Section IV discusses the experimental validation of both approaches and critically evaluates the obtained results.

\section{EXPERIMENTAL SETUP}

\section{A. Design of the badminton robot}

Fig. 1 shows a schematic representation of the considered system. A detailed description of the robot and its subsystems can be found in [13]. The robot has 3 degrees of freedom a linear axis, a rotational axis and a hit axis. The linear axis is used to position the robot across the field using a linear motor. As this axis is the main energy consumer, its motion is subject to optimization in this paper. The dynamics between the current to the linear motor and its position is close to a double integrator.

A stereo camera system is used to detect the shuttle cock. Based on shuttlecock location measurements, the shuttle cock trajectory is estimated. Interception logic determines the appropriate hit time and the robot configuration (i.e. hit angle, rotational angle and position along the linear axis) in order to hit back the shuttle to the human opponent at a point along the predicted shuttlecock trajectory.

As the shuttlecock trajectory is known with a large uncertainty immediately after the human player has hit the 


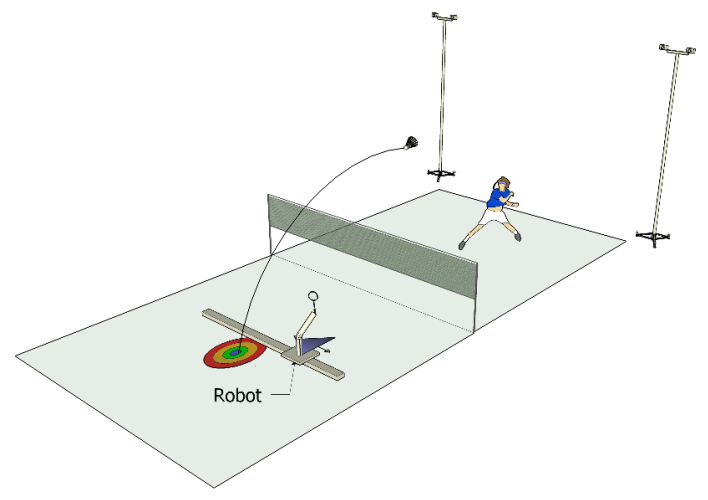

Fig. 1. Concept of the robot playing field and the visual system

shuttlecock, the interception point (position and time) estimation is repeated continuously throughout the motion. Since these estimates can change drastically during the motion, the developed control algorithms must be able to deal with continuously changing interception points.

\section{B. Proximate time-optimal servo (PTOS)}

The total time available for the badminton motion is limited by the expected fly-time of the badminton shuttlecock (usually less than $1 \mathrm{sec}$ ). For this reason, originally, the time-optimal control approach PTOS [2] was implemented on the linear axis, which aims to position the robot in the hit configuration as fast as possible taking into account actuator constraints. Since the PEOS approach discussed in section III is based on PTOS, this algorithm is discussed first. For the considered ideal double integrator plant dynamics (with the position $y$ as output and $u$ equal to the acceleration $a$ as input)

$$
\begin{aligned}
& \dot{y}(t)=v(t) \\
& \dot{v}(t)=a(t)=u(t)
\end{aligned}
$$

where the acceleration $a(t)$ and velocity $v(t)$ are bounded $(-\hat{a} \leq a(t) \leq \hat{a}$, and $-\hat{v} \leq v(t) \leq \hat{v})$, an approximation of the time-optimal control is relatively simple and easy to implement. If the goal is to move from $y=0$ to a desired position $y=y_{\text {des }}$, the control signal $u$ has the form

$$
\begin{aligned}
u(t) & =\hat{a} \operatorname{sat}_{1}\left(k_{2}\left(\operatorname{sat}_{\hat{v}}\left(f_{\text {ptos }}(e(t))\right)-v(t)\right)\right) \\
e(t) & =y_{\text {des }}-y(t)
\end{aligned}
$$

where

$$
\begin{aligned}
& f_{\text {ptos }}(e(t))= \begin{cases}\operatorname{sgn}(e(t))(\sqrt{2 \hat{a} \alpha|e(t)|}-J), & |e(t)|>y_{l} \\
\frac{k_{1}}{k_{2}} e(t), & |e(t)| \leq y_{l}\end{cases} \\
& k_{1}=\frac{2 \alpha \xi^{2}}{y_{l}}, k_{2}=2 \xi^{2} \sqrt{\frac{2 \alpha}{\hat{a} y_{l}}}, J=\frac{1}{\sqrt{\frac{2}{\hat{a} \alpha y_{l}}}} \\
& \operatorname{sat}_{x}(x)=\left\{\begin{array}{lc}
-x, & x<x \\
x, & x>x \\
x, & \text { otherwise }
\end{array}\right.
\end{aligned}
$$

The controller parameters $k_{1}, k_{2}, J$ depend on the design parameters $y_{l}, \xi$, $\alpha$ which can be used to gain robustness at the cost of small loss of time optimality. $y_{l}$ is the size of the region near the target $y_{d e s}$, where the PTOS algorithm behaves like a linear controller. In the linear region $\xi$ is the desired damping of the closed loop system. $\alpha \leq 1$ is a discount factor to obtain a safety margin during the deceleration.

PTOS can be implemented as a feedback controller or as a trajectory and feedforward generator [2]. In this work PTOS is used as a trajectory and feedforward generator, in combination with a position feedback controller with a bandwidth of $10 \mathrm{~Hz}$ running at a sampling rate of $1 \mathrm{kHz}$. This feedback controller, further referred to as tracking controller, guarantee stable and accurate positioning. The trajectory and feedforward signals are obtained by closing the PTOS loop on a double integrator model plant, instead of the real plant. The obtained plant model output and input are applied as position reference and feedforward for the tracking controller. Both the PEOS and EOMPC approaches discussed below are used in this configuration, i.e. are used in combination with a tracking controller.

\section{The robot energy consumption}

An energy consumption model has been identified experimentally. First, current and voltage measurements for a set of typical motions were performed at the entry of the electrical cabinet of the robot. Estimates of the powers were obtained by multiplying these voltages and currents.

Then a parametric model was fitted on these data using as inputs robot velocity $(v)$ and acceleration $(a)$ estimates obtained from the position data measured during the above mentioned experiments. The following regression model was obtained.

$$
P=160.26+24.13 \times v^{2}+18.35 \times a v+1.57 \times a^{2}
$$

The structure of this model is determined based on the following physical considerations: (i) $a^{2}$ relates to the resistive losses, (ii) $v^{2}$ relates to the viscous friction losses and (iii) $a v$ relates to the mechanical power. This model is used in the PEOS and EOMPC approaches.

\section{PRoposed METHOdS}

\section{A. PEOS with Genetic Algorithm (GA) tuning}

The PTOS algorithm [14] is the basis of the proximate energy-optimal servo (PEOS) approach. For time-optimal point-to-point motions, the PTOS algorithm generates the control signal $u(t)$ assuming a constant maximum acceleration $\hat{a}$ and velocity $\hat{v}$ determined by the physical limitations of the linear motor. The idea in the developed energy-optimal approach is to adapt these parameters during the motion of the badminton robot in order to increase the energy efficiency. According to Eq. (3) an effective method to improve energy efficiency, is to reduce the velocity and acceleration during the motion of the robot. This can be achieved by limiting the parameters $\hat{a}$ and $\hat{v}$ in the PTOS algorithm. As a consequence, 


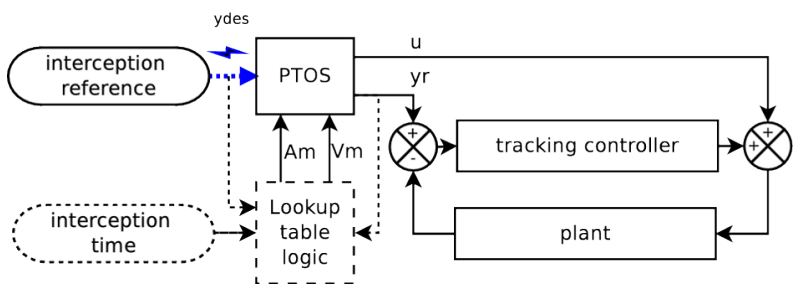

Fig. 2. PEOS motion controller structure

the time needed to reach the interception point will increase such that the risk occurs that the robot arrives too late to intercept and hit back the shuttlecock. Therefore, a trade-off between the energy consumption and the motion time has to be determined. For this purpose look-up-tables are introduced in the control scheme on Fig. 2. While in the original scheme $\hat{a}$ and $\hat{v}$ are constant, in the new scheme in Fig. 2 the interception point and time are processed by look-up tables in order to modify $\hat{a}$ and $\hat{v}$. Since the fundamental structure of the motion controller is preserved and no significant increase of the online computational complexity is introduced by the proposed modification, the PEOS algorithm can be applied on the same limited embedded hardware as the baseline PTOS controller. The look-up-tables, which are used to determine $\hat{a}$ and $\hat{v}$ are created according to the following procedure:

1) Multi-objective tuning of $\hat{a}$ and $\hat{v}$ in simulation: The selection of optimal values for $\hat{a}$ and $\hat{v}$ (lookup table in Fig. 2) is a multi-objective optimization problem, where the optimal trade-off is searched between the consumed energy during a motion, the motion duration and the travelled distance. This problem is solved using a multi-objective Genetic Algorithm (GA). Each individual of a generation of the GA is parametrized by a chromosome containing a different triplet $\left(\hat{a}, \hat{v}, y_{\text {des }}\right)$. Using numerical simulations, for a given desired distance $y_{\text {des }}$ of the robot's linear motion and for a given maximum acceleration $\hat{a}$ and maximum velocity $\hat{v}$ in the PTOS algorithm, the values of the time $T$ and energy $E$ are calculated.

The simulation function is implemented as a PTOS controller (2) driving an ideal double-integrator plant (1), and provides the acceleration and velocities during the motion time $T$. Eq. (3) is used to calculate the dissipated power, which is integrated to obtain the consumed energy $E$. The cost function used to minimize the values in the sense of Pareto has the following form

$$
\left(\hat{a}, \hat{v}, y_{\text {des }}\right) \stackrel{\text { cost }}{\longrightarrow}\left(T, E, \frac{1}{y_{\text {des }}}\right)
$$

This function calls internally the simulation function and uses the values of $T$ and $E$. The fitness of each individual is determined based on the multi-objective cost triplet $\left(E, T, 1 / y_{\text {des }}\right)$, where $E$ and $T$ are obtained using the simulation function. A multi-objective genetic algorithm was run for 50 generations, each of 200 individuals with the cost function (4)
2) Constructing the $\hat{a}$ and $\hat{v}$ look-up tables: For each point $\left(E, T, y_{d e s}\right)$ on the final Pareto front, the corresponding acceleration and velocity values from the chromosome triplet $\left(\hat{a}, \hat{v}, y_{\text {des }}\right)$ are also known. This permits us to construct the following functional mappings

$$
\begin{aligned}
\left(T, y_{\text {des }}\right) & \mapsto \hat{a} \\
\left(T, y_{\text {des }}\right) & \mapsto \hat{v},
\end{aligned}
$$

which can be used to determine the PTOS settings in an energy-optimal way. The functional mappings were approximated using a piece-wise linear fit according to the following procedure:

1) The $\left(T, y_{\text {des }}\right)$-plane is divided into a grid, consisting of all combinations from the following sets $T \in\{0.75,1,1.25,1.5\}, \quad y_{\text {des }} \in$ $\{0.4,0.6,0.8,1,1.25,1.5,1.75,2,2.25\}$.

2) For each point of the grid, the 50 closest points from the Pareto front are selected with the corresponding PTOS parameters $\hat{a}$ and $\hat{v}$.

3) A linear surface fit is performed on these 50 points one for the $\left(T, y_{\text {des }}\right) \mapsto \hat{a}$ relationship and another for $\left(T, y_{\text {des }}\right) \mapsto \hat{v}$, resulting in linear approximating coefficients.

4) Two look-up tables are then build consisting of an evaluation of the local linear approximations on the grid points. They are shown on Fig. 3 and Fig. 4.

The above procedure takes some time, but it is to be performed only once off-line. The resulting $\left(T, y_{\text {des }}\right) \mapsto \hat{a}$ and $\left(T, y_{\text {des }}\right) \mapsto \hat{v}$ approximations stored in the form of look-up tables are easy and quick to evaluate on-line.

\section{B. Energy-Optimal MPC}

Energy-optimal MPC (EOMPC) [15] is a control method that aims at performing energy-optimal point-to-point motions within a given motion time. EOMPC solves this optimal control problem in real-time taking into account system constraints. Because of the real-time solution strategy, interception reference and interception time can be changed during the motion. Energy optimality is achieved by setting the object function of the MPC optimization problem equal to the system's energy consumption. EOMPC is essentially using the time-optimal MPC (TOMPC) approach described in [16] which minimize the point-to-point motion time of linear time-invariant systems while taking into account the system constraints. The motion time minimization of EOMPC is stopped once the requested interception time is reached. As a result, the motion will be executed in the given interception time, and not faster, and the energy consumption will be minimized. If for some reason the requested motion time is too short for the considered system and displacement considering the system constraints, EOMPC approach will perform exactly the same as TOMPC - in this case it will automatically find the shortest possible motion time (which obviously will be larger than the requested motion time) and realize the motion in an time-optimal way. 


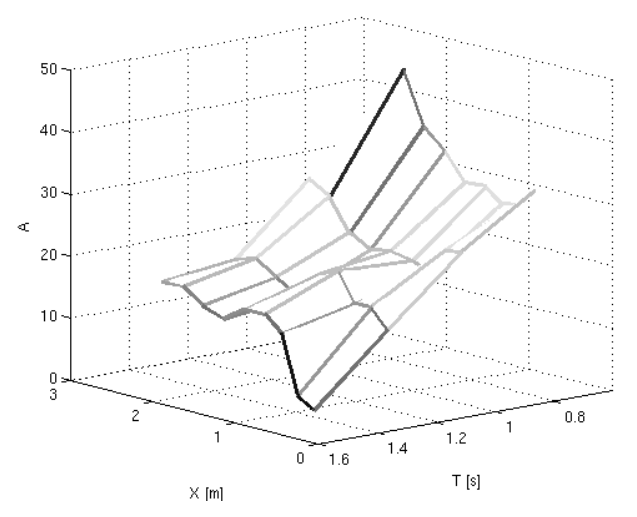

Fig. 3. Maximum acceleration look-up-table $\left(T, y_{\text {des }}\right) \mapsto \hat{a}$

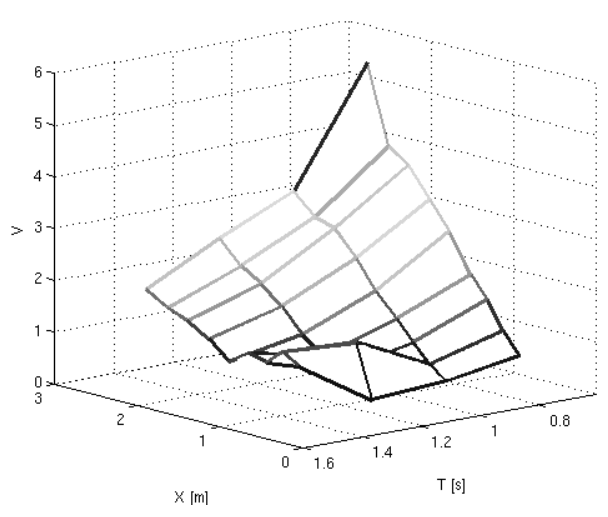

Fig. 4. Maximum velocity look-up-table $\left(T, y_{\text {des }}\right) \mapsto \hat{v}$

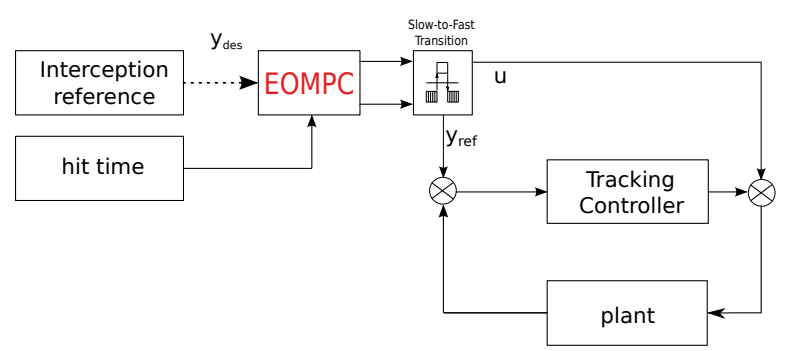

Fig. 5. EOMPC motion controller structure

The EOMPC generates position reference $y_{\text {ref }}$ and acceleration control signals $u$, assuming the double integrator model (1), which are applied to the position feedback controlled linear motor, as shown in Fig.5. This position feedback controller (indicated in Fig.5 as tracking controller) runs at a sampling rate of $1 \mathrm{kHz}$. The sampling rate of the EOMPC is limited to $100 \mathrm{~Hz}$ due to the computational complexity of the MPC real-time optimization. Due to this sampling rate difference, a zero-order-hold extrapolation is performed on the EOMPC control signals as indicated in Fig.5.

The EOMPC optimization problem is formulated as a twolayer optimization problem. First the settling time defined as the number $N$ of discrete time instants required for the system to be at rest at the desired set point is determined based on the available motion time $K^{*} . K^{*}$ depends on the interception time provided by the interception logic. Once $N$ is determined, the optimal signal sequence is obtained by solving the MPC optimization problem. Hence, at each time instance $l$ the following two optimization problems, 'Problem A' and 'Problem B', are to be solved:

'Problem A', denoted as $P_{A}\left(\hat{\mathbf{x}}_{l}, N\right)$, calculating the energy optimal control signal for a given $N$ (which is obtained by solving 'Problem B') while respecting the system constraints, is defined as follows:

$$
\begin{gathered}
V_{A}^{*}=\min _{\mathbf{u}} E_{\text {loss }}(\mathbf{u}) \\
\text { s.t. } \quad \mathbf{x}_{0}=\hat{\mathbf{x}}_{l} \\
\mathbf{x}_{k+1}=\mathbf{A}_{k} \mathbf{x}_{k}+\mathbf{B}_{k} u_{k}, \quad k=0,1, \cdots, N_{\text {max }}-1 \\
y_{k}=\mathbf{x}_{k}(1), \quad k=0,1, \cdots, N_{\text {max }} \\
v_{k}=\mathbf{x}_{k}(2), \quad k=0,1, \cdots, N_{\text {max }} \\
v_{\text {min }}, u_{\text {min }}, y_{\text {min }} \leq v_{k}, u_{k}, y_{k} \leq v_{\text {max }}, u_{\text {max }}, y_{\text {max }} \\
y_{k}=y_{\text {des }}, \quad k=N, \cdots, N+n-1 \\
u_{k}=0, \quad k=N, \cdots, N_{\text {max }}-1
\end{gathered}
$$

In Equation (6), $\mathbf{u}=\left[u_{0}, \ldots, u_{N_{\max }-1}\right]$ is the optimization variable over the prediction horizon $N_{\max } . \hat{\mathbf{x}}_{l}$ in Eq. $(6 \mathrm{~b})$ is the system state at time instance $l$. Since the EOMPC is used here in an open-loop configuration generating reference position and acceleration feed forward, $\hat{\mathbf{x}}_{l}$ is obtained through openloop simulation of the double integrator system model (1). The optimization is based on the system model Eq. (6c). The two state variables are the position and velocity as shown in Eq. (6d-6e). Eq. (6f) specify the constraints on the position, the velocity, and the acceleration of the system. Eq. $(6 \mathrm{~g}-6 \mathrm{~h})$ are moving endpoint constraints and impose the system to be at rest at the desired setpoint $y_{\text {des }}$ at time $N$.

The system dynamic matrices $\mathbf{A}_{k}$ and $\mathbf{B}_{k}$ are time dependent because of the non-equidistant sampling time, which is called 'Blocking' [15]. Blocking is utilized in the EOMPC in order to be able to extend the prediction horizon to 2 seconds such that all possible requested displacements can be reached within the interception time, while limiting the number of optimization variables $N_{\max }=35$. Equidistant sampling at a sampling rate of $100 \mathrm{~Hz}$ in combined with a prediction horizon of 2 seconds would result in 200 optimization variables and yield an optimization problem that is too large to be solved in real time at this specific sampling rate.

'Problem A' defines an admissible set

$$
\mathbf{X}(N)=\left\{\hat{\mathbf{x}}_{l} \mid P_{A}\left(\hat{\mathbf{x}}_{l}, N\right) \text { is feasible }\right\}
$$

$\mathbf{X}(N)$ in (7) is the set of system states from which the set point can be reached within $N$ time steps, while respecting all system constraints Eq. (6f).

'Problem B', calculating the settling time $N$, is defined as follows:

$$
\begin{aligned}
V_{B}^{*}\left(\hat{\mathbf{x}}_{l}, K^{*}\right) & =\min N \\
\text { s.t. } \quad \hat{\mathbf{x}}_{l} & \in \mathbf{X}(N) \\
\max \left(N_{\min }, K^{*}\right) & \leq N \leq N_{\max }
\end{aligned}
$$


In Equation (8), $N$ is bounded by $N_{\max }$ and the maximum of $N_{\min }$ and $K^{*}$. To guarantee unconstrained solvability, $N_{\min }$ should be at least $n$ with $n$ the number of states [17]. Hence, at each time step $l$, 'problem B' minimizes $N$ up to $K^{*}$ if $y_{d e s}$ can be reached in $K^{*}$ time steps $\left(P_{A}\left(\hat{\mathbf{x}}_{l}, K^{*}\right)\right.$ is feasible) except if (i) $y_{\text {des }}$ can be reached in less than or equal to $N_{\min }$ time steps with $K^{*}<N_{\text {min }}$, yielding $N=N_{\text {min }}$ or if (ii) $y_{\text {des }}$ can't be reached in $K^{*}$ time steps, yielding $N>K^{*}$.

The object function $E_{\text {loss }}$ is based on the experimentally identified energy consumption model Eq. (3) which considers both copper and friction losses. This energy consumption model is an extension of the model considered in [15], where only copper losses are taking into account. Based on equation (3), the object function of the EOMPC (6a) can be formulated as follows:

$$
\begin{aligned}
E_{\text {loss }}=\mathbf{v}^{\mathrm{T}} \mathbf{R}_{\mathbf{1}} \mathbf{v} & +\mathbf{u}^{\mathrm{T}} \mathbf{R}_{\mathbf{2}} \mathbf{v}+\mathbf{u}^{\mathrm{T}} \mathbf{R}_{\mathbf{3}} \mathbf{u} \\
\mathbf{R}_{\mathbf{1}} & =c_{1} * \mathbf{I}_{\mathbf{r}} \\
\mathbf{R}_{\mathbf{2}} & =c_{2} * \mathbf{I}_{\mathbf{r}} \\
\mathbf{R}_{\mathbf{3}} & =c_{3} * \mathbf{I}_{\mathbf{r}}
\end{aligned}
$$

with $c_{1}=24.13, c_{2}=18.35$ and $c_{3}=1.57$, the parameters of the identified energy model Eq. (3). $\mathbf{v}=\left[v_{0}, \ldots, v_{N_{\max }-1}\right]$ with velocity $v_{k}\left(k=0, \cdots, N_{\max }-1\right)$ given by Eq. (6e). $\mathbf{I}_{\mathbf{r}} \in \mathbf{R}^{N_{\max } * N_{\max }}$ is a diagonal matrix but not an identity matrix because of the non-equidistant sampling time considered in the prediction horizon. The $i^{\text {th }}$ diagonal element $\left(i=1, \cdots, N_{\text {max }}\right)$ equals the duration of the $k^{\text {th }}$ discrete time interval divided by the sampling time $T_{s}=0.01[s]$. An advantage of EOMPC is obviously shown here that different models of the energy losses can be easily dealt with by defining different weighting matrices $\mathbf{R}_{\mathbf{1}}, \mathbf{R}_{\mathbf{2}}$ and $\mathbf{R}_{\mathbf{3}}$.

This optimization problem is a convex QP. Its size is constant and hence independent of the distance between the robot and the target position. It is solved by the on-line active set method called qpOASES [10].

\section{EXPERIMENTAL RESULTS}

The two proposed control schemes were implemented on the real badminton robot hardware and tested. It is difficult to compare the performance and energy-efficiency of the robot during a real badminton game because the same series of the motions can never be repeated exactly. Therefore, a standard test motion of approximately 10 minutes was recorded and repeated twice on the robot, once using the PEOS and once using the EOMPC approach. A total of 84 shuttle hits are performed during the play.

Keeping in mind our original multi-objective formulation, the following criteria are evaluated:

- the energy consumed for the series of motions

- the on-time arrival of the robot at the interception points

A representative zoom of part of the results are shown in Fig. 6. Remark that the interception time shown in Fig. 6(a) does not necessarily change linearly with time and the interception reference shown in Fig. 6(b) changes during motion. (a)

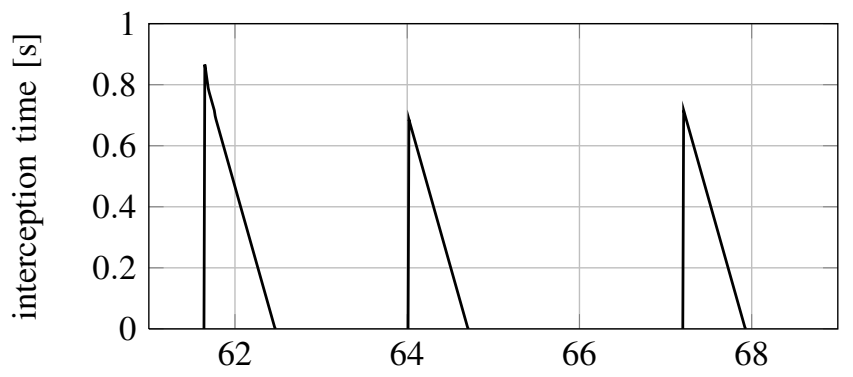

(b)

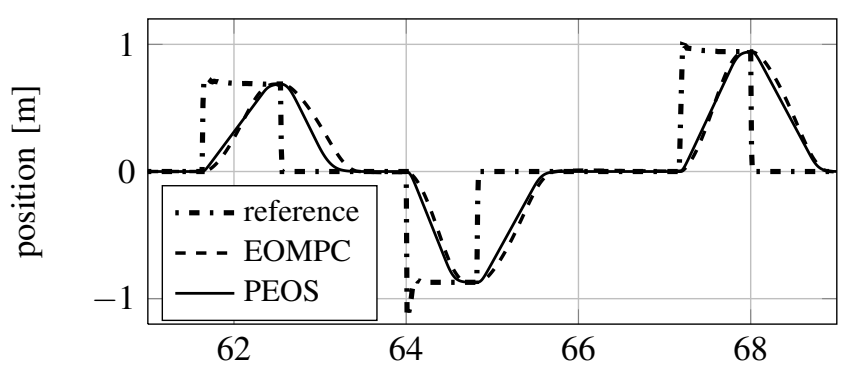

(c)

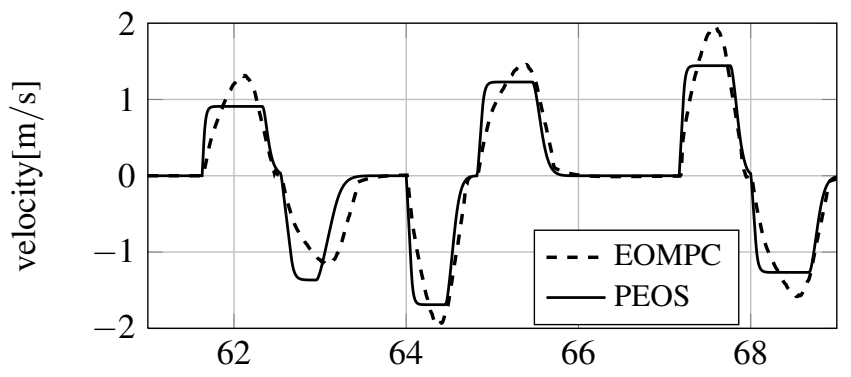

(d)

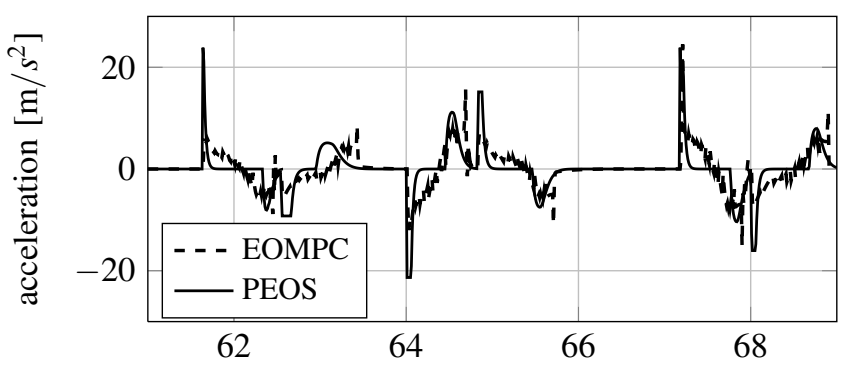

$(e)$

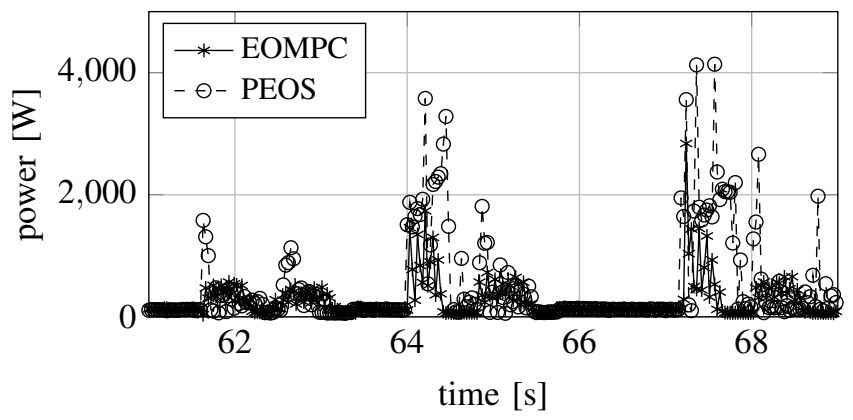

Fig. 6. (a) Interception time (b) Motor positions (c) Motor velocity (d) Motor acceleration (e) Motor power 


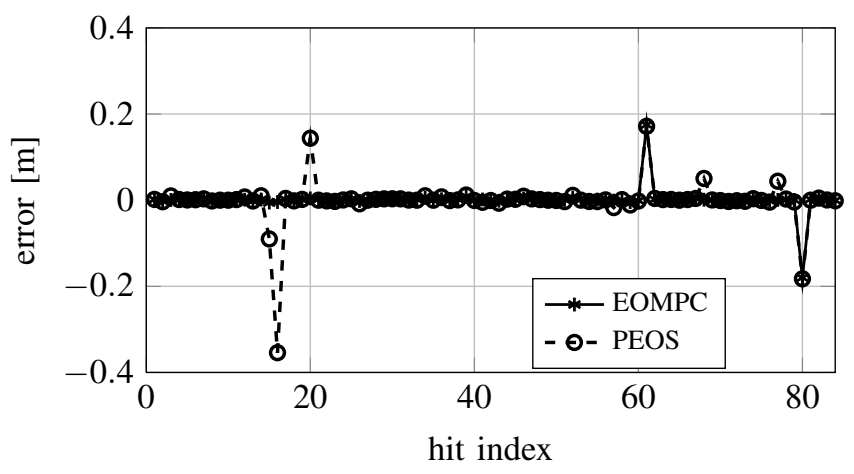

Fig. 7. Interception errors at hit moment

This is because the interception logic continuously updates the interception point as explained in section II. Once the robot has hit the shuttle, it moves back to the center of the linear motor within a given constant homing time and waits for the next desired interception reference requested.

The EOMPC requires less energy than PEOS. For the experiment discussed above, EOMPC and PEOS consume $126 k J$ and $134 k J$ respectively. Fig. 7 shows the positioning error, that is the difference between the actual position at the specified hit time and the interception point, for each of the 84 requested shuttle hits. Due to the high bandwidth of the position feedback controller, the positioning error is mainly caused by the reference generating (PEOS and EOMPC) algorithms. A positioning error larger than $3 \mathrm{~cm}$ is considered as a missed hit. Fig. 7 clearly shows that the EOMPC ( 2 missed hits) performs better than the PEOS approach ( 7 missed hits). This is due to the fact that the EOMPC can make a good tradeoff between the energy consumption and the positioning error which can not be done by PEOS. For each motion, when the required motion time is larger than that of time-optimal MPC, EOMPC performs energy optimal motion, otherwise, EOMPC performs time optimal motion regardless energy consumption.

In order to compare these two proposed energy-optimal control schemes (EOMPC and PEOS) with the time-optimal PTOS, the PTOS was implemented on the real badminton robot hardware for the same test. PTOS consumes $229 \mathrm{~kJ}$ and 3 missed hits. A huge reduction of the energy, about $40 \%$, is obtained by using EOMPC and PEOS comparing with PTOS. EOMPC and PTOS have the same positioning error.

\section{CONCLUSiON}

In this paper two approaches for energy optimal motion are presented. The first approach is based on a classical and mature industrial technology, namely PTOS. It is tuned using genetic algorithm with the aim to improve the energy efficiency of the motion. The second approach EOMPC is based on modelpredictive control and includes optimization on each time-step of the cost function based on actual energy of the robot.

Both approaches show very good results. A strong reduction of the dissipated energy is obtained compared to time-optimal control while the performance degradation is negligible. EOMPC outperforms PEOS - both in energy and in precision because the EOMPC can make a good trade-off between the energy consumption and the positioning error. On the other hand, the PEOS approach is more simple and requires less performance hardware to implement. The EOMPC approach also relies on the availability of cost function describing the energy in a quadratic form, which is probably not possible for all systems.

Future work can apply the approach to higher dimensional systems with more flexible modes. In this case EOMPC, which can deal with high dimension systems, have a very strong advantage with respect to PEOS. Online estimation of the energy cost of the system and corresponding update of the optimization coefficients in the EOMPC could also be another interesting extension.

\section{REFERENCES}

[1] M. L. Workman, "Adaptive proximate time-optimal servomechanisms," Ph.D. dissertation, Stanford Univ., Stanford, CA, 1987.

[2] A. Dhanda and G. F. Franklin, "An improved 2-dof proximate time optimal servomechanism," IEEE Transactions on magnetics, vol. 45, no. 5, pp. 2151-2164, 52009.

[3] B. G. Dijkstra, N. Rambaratsing, C. Scherer, O. H. Bosgra, M. Steinbuch, and S. Kerssemakers, "Input design for optimal discrete time point-to-point motion of an industrial xy-positioning table," in Conference on Decision and Control, 12 2000, pp. 901-906.

[4] V. Kluchev, Electric drive theory. Enegroatomizdat, 2001.

[5] E. S. Sergaki, G. S. Stavrakakis, and A. D. Pouliezos, "Optimal robot speed trajectory by minimization of the actuator motor electromechanical losses," J. Intell. Robotics Syst., vol. 33, no. 2, pp. 187-207, Feb. 2002.

[6] B. Cao, G. Dodds, and G. Irwin, "Constrained time-efficient and smooth cubic spline trajectory generation for industrial robots," Control Theory and Applications, IEE Proceedings -, vol. 144, no. 5, pp. $467-475$, sep 1997.

[7] E. Camacho and C. Bordons, Model Predictive Control, ser. Advanced Textbooks in Control and Signal Processing. Springer, 2004.

[8] J. Maciejowski, Predictive Control: With Constraints, ser. Pearson Education. Prentice Hall, 2002.

[9] M. Kvasnica, Real-Time Model Predictive Control Via Multi-Parametric Programming: Theory and Tools. VDM Verlag, 2009.

[10] H. J. Ferreau, H. G. H.G. Bock, and M. Diehl, "An online active set strategy to overcome the limitations of explicit MPC," International Journal of Robust and Nonlinear Control, vol. 18, no. 8, pp. 816-830, 2008.

[11] R. Steuer, Multiple criteria optimization: theory, computation, and application, ser. Wiley-Interscience series in systems and optimization. Wiley, 1986.

[12] K. Deb, Multi-objective optimization using evolutionary algorithms, ser Wiley-Interscience series in systems and optimization. John Wiley \& Sons, 2001.

[13] J. Stoev, A. Bartic, S. Gillijns, and W. Symens, "Badminton playing robot - a multidisciplinary test case in mechatronics," in 5th IFAC Symposium on Mechatronic Systems, Marriott Boston Cambridge, USA, sept 2010.

[14] J. Stoev, G. Pinte, and W. Symens, "Time-constrained energy-optimal motion control - application to a badminton robot," in The 13th Mechatronics Forum International Conference, Linz, Austria, 92012.

[15] X. Wang, J. Stoev, G. Pinte, and J. Swevers, "Energy optimal point-topoint motion using model predictive control," in 5th annual Dynamic Systems and Control Conference (DSCC), Ft. Lauderdale, FL, USA, 10 2012.

[16] L. Van den Broeck, M. Diehl, and J. Swevers, "A model predictive control approach for time optimal point-to-point motion control," Mechatronics, vol. 21(7), pp. 1203-1212, 2011.

[17] T. Kailath, "Linear systems," Prentice Hall, 1980. 